
\title{
25 Research Soure \\ TMEM205 Reduces the Sensitivity of Gastric Cancer to Cisplatin Through TAM/M2 Polarization
}

\section{Xuwei Wu}

Yanbian University Medical College

\section{Ziqi Meng}

Yanbian University Health Science Center: Yanbian University Medical College

\section{Rui Zhang}

Yanbian University Medical College

\section{Meihua Zhang}

Yanbian University Hospital

\section{Zhengri Piao}

Yanbian University Hospital

Tiefeng Jin ( $\nabla$ jintf@ybu.edu.cn )

Yanbian University https://orcid.org/0000-0001-8957-696X

\section{Research Article}

Keywords: gastric cancer, transmembrane protein 205, cisplatin resistance, tumor-associated macrophages, Wnt/ $\beta$-catenin

Posted Date: March 2nd, 2022

DOI: https://doi.org/10.21203/rs.3.rs-1381798/v1

License: (c) (1) This work is licensed under a Creative Commons Attribution 4.0 International License.

Read Full License 


\section{Abstract}

Cisplatin (DDP) is a basic chemotherapy drug for gastric cancer (GC). With the increase of DDP drug concentration in clinical treatment, cancer cells gradually became resistant. Therefore, it is necessary to find an effective therapeutic target to enhance the sensitivity of GC to DDP. Transmembrane protein 205 (TMEM205) plays significant roles in DDP resistance, but the specific mechanism of TMEM205 in DDPresistant GC has not been studied. Databases showed that TMEM205 overexpresses in GC. In vitro results showed that TMEM205 enhances proliferation, stemness, epithelial-mesenchymal transition (EMT) process, migration and angiogenesis of SGC-7901/DDP cells by activating the Wnt/ $\beta$-catenin signaling pathway. In addition, TMEM205 promotes GC progression by inducing M2 polarization of tumor-associated macrophages (TAMs). These results suggest that TMEM205 may be an effective target to regulate the sensitivity of GC to DDP, providing a new therapeutic direction for clinical treatment.

\section{Introduction}

GC is the fifth and fourth highest malignant tumor in the world in terms of morbidity and mortality ${ }^{[1]}$. For the treatment of GC, DDP is one of the chemotherapy drugs for patients with postoperative and advanced, but its drug resistance affects the treatment effect ${ }^{[2]}$. Therefore, it is necessary to find an effective therapeutic target to enhance the sensitivity of GC cells to DDP.

As a member of the transmembrane proteins (TMEMs), TMEM205 constructed transmembrane channels for a variety of substances, mediating communication between the intracellular and extracellular environment ${ }^{[3]}$. TMEM205 overexpressed in DDP-resistant human epidermoid cancer cells and related to chemical resistance. In addition, TMEM205 underwent nuclear translocation in DDP-resistant cells, and interacted with RAB8 to promote the accumulation of DDP outside the cell, enhancing DDP resistance ${ }^{[4,5]}$. Related studies have shown that TMEMs were involved in cancer progression by promoted cancer cells proliferation, stemness, migration, angiogenesis, and EMT process. TMEM107 affected the proliferation, migration, and EMT process of NSCLC cells ${ }^{[6]}$. Knocking down TMEM215 inhibited the angiogenesis of HUVECs $^{[7]}$. TMEM119 enhanced stemness of breast cancer cells by activating the Wnt/ $\beta$-catenin signaling pathway ${ }^{[8]}$. However, the effects of TMEM205 on GC stemness, migration, angiogenesis and EMT process are unclear.

Tumor-associated macrophages are the most abundant immune cells in tumor microenvironment (TME). TAMs can be polarized into classically activated M1 type (TAM/M1) and alternative activated M2 type (TAM/M2) under the stimulation of different environment and inducing factors ${ }^{[9]}$. TAM/M2 polarization enhanced the resistance of GC to DDP, leading to treatment failure ${ }^{[10]}$. Yes associated protein 1 enhanced tumor cells resistance to 5 -fluorouracil through inducing TAM/M2 polarization ${ }^{[11]}$. Regulation of TAMs polarization may affect cancer progression and chemotherapy resistance. However, the relationship of TMEM205, TAMs polarization, and GC progression had not been reported. 
In this study, we explored the effects of TMEM205 on the proliferation, stemness, migration and EMT process of SGC-7901/DDP cells, clarified the molecular mechanism of TMEM205 inhibiting DDP-resistant GC cells by regulating TAMs polarization, and providing theoretical basis for clinical treatment of DDP8resistant GC.

\section{Materials And Methods 2.1 Cell lines}

The human normal gastric epithelial cells (GES-1) and the human GC cells (SGC-7901) were obtained from the Cancer Research Center of Yanbian University. The DDP-resistant GC cells (SGC-7901/DDP) and THP-1 cells were purchased from Shanghai Fuheng company. All cells were cultured in RPMI-1640 medium containing $10 \% \mathrm{FBS}$ and $1 \%$ penicillin-streptomycin and at $37{ }^{\circ} \mathrm{C}, 5 \% \mathrm{CO}_{2}$.

\subsection{Transfection}

We purchased TMEM205 specific siRNA from RIBOBIO (Guangzhou, China), including si-TMEM205-1, siTMEM205-2, si-TMEM205-3 and siRNA (si-Con). 1 $\times$ buffer, siRNA and dye transfer solution were mixed and incubated at RT for 20 min, and added to penicillin streptomycin-free RPMI-1640 cultured SGC7901/DDP cells for $48 \mathrm{~h}$.

\subsection{Established co-cultured system}

Cells $\left(1 \times 10^{5} /\right.$ well) were inoculated in upper chamber of transwell device (BD Biosciences, Piscataway, NJ, USA). THP-1 cells were induced to macrophages and inoculated in lower chamber. Added $1.46 \mu \mathrm{g} / \mathrm{mL}$ DDP to the corresponding wells. After $48 \mathrm{~h}$, THP-1 cells were detected by western blot, immunofluorescence and flow cytometry assays.

\subsection{Western blot assay}

Cells were lysed with appropriate RIPA buffer containing appropriate protease and phosphatase inhibitor (CoWin Biosciences, Beijing, China). Protein concentration was measured by BSA Protein Assay Kit (Roche, Basel, Switzerland). Denatured proteins were separated by SDS-PAGE glue and transferred to PVDF membranes (Millipore, Billerica, MA, USA). PVDF membranes were incubated with corresponding primary antibody overnight at $4{ }^{\circ} \mathrm{C}$. Next day, PVDF membranes were incubated with corresponding secondary antibody at RT for $1 \mathrm{~h}$. The bands were obtained using the Gel Imaging System (Bio-Rad, Hercules, CA, USA).

\subsection{MTT assay}

Cells $\left(1 \times 10^{3} /\right.$ well) were inoculated in $96-$ well plates, and treated with different concentration of DDP $(0$, $0.4,0.8,1.2,1.6,2 \mu \mathrm{g} / \mathrm{mL})$ for $48 \mathrm{~h} .100 \mu \mathrm{L}$ MTT $(1 \mathrm{mg} / \mathrm{mL})$ was added to each well for $4 \mathrm{~h}$ at $37^{\circ} \mathrm{C}$. Absorbance value was measured at $490 \mathrm{~nm}$ after adding $100 \mu \mathrm{L}$ DMSO to each well.

\subsection{Clone formation assay}


Cells (500/well) were inoculated in 6-well plates for 14 days. Cells were fixed with $4 \%$ paraformaldehyde for 30 min, washed with PBS, and stained with hematoxylin at RT. The number of colony formation was analyzed.

\subsection{EDU assay}

SGC-7901/DDP cells $\left(5 \times 10^{4} /\right.$ well) were inoculated in 96-well plates, and corresponding wells were treated with $1.46 \mu \mathrm{g} / \mathrm{mL}$ DDP for $48 \mathrm{~h}$. $100 \mu \mathrm{L}$ EDU medium was added to each well for $2 \mathrm{~h}$ at $37{ }^{\circ} \mathrm{C} .4 \%$ paraformaldehyde fixed cells for $30 \mathrm{~min} .50 \mu \mathrm{L}$ glycine $(2 \mathrm{mg} / \mathrm{mL})$ incubated cells for $5 \mathrm{~min} .100 \mu \mathrm{L} 0.5 \%$ Triton X-100 (CWBIO Biosciences) permeabilizing agent incubated cells for $5 \mathrm{~min}$. Apollo staining reaction solution incubated cells at RT for $30 \mathrm{~min}$. Permeabilizing agent to decolorize cells and methanol to wash cells. $1 \times$ Hoechst33342 reaction solution incubated cells for $30 \mathrm{~min}$ at RT. Using inverted microscope (NIH, Bethesda, MD, USA) to take pictures.

\subsection{Wound healing assay}

SGC-7901/DDP cells were inoculated in 6-well plates. The cells were scratched with $200 \mu \mathrm{L}$ tips until fused to $80 \% .1 .46 \mu \mathrm{g} / \mathrm{mL}$ DDP was added into corresponding wells. The images were obtained at $0 \mathrm{~h}, 24$ $\mathrm{h}$ and $48 \mathrm{~h}$ using an inverted microscope.

\subsection{Transwell assay}

SGC-7901/DDP cells $\left(1 \times 10^{5} /\right.$ well) were inoculated into upper chamber of transwell device (BD Biosciences, Piscataway, NJ, USA), and RPMI-1640 complete medium was added into the lower chamber. $1.46 \mu \mathrm{g} / \mathrm{mL}$ DDP was added to corresponding wells. Cells fixed with $4 \%$ paraformaldehyde and stained with hematoxylin after $48 \mathrm{~h}$. Cells were counted under a microscope.

\subsection{Immunofluorescence (IF) assay}

Cells were inoculated in 6-well plates. $1.46 \mu \mathrm{g} / \mathrm{mL}$ DDP was added to corresponding wells and cultured for $48 \mathrm{~h}$ at $37^{\circ} \mathrm{C}$. Cells fixed with $4 \%$ paraformaldehyde and permeabilized with $0.5 \%$ Triton-X-100. Cells blocked with $3 \%$ BSA (Solarbio, Beijing, China) for $2 \mathrm{~h}$ at RT. Primary antibodies incubated cells at $4{ }^{\circ} \mathrm{C}$ overnight. Primary antibodies include E-cadherin (1:200), Vimentin (1:100), $\beta$-catenin (1:100), CD86 (1:200) (Santa Cruz Biotechnology, Dallas, TX, USA), CD206 (1:200), Oct4 (1:100), CD44 (1:100) (Cell Signaling Technology, USA). Next day, cells were incubated with secondary antibodies for $2 \mathrm{~h}$ at RT, then counterstained with DAPI (Solarbio, Beijing, China). Using LeicaSP5II confocal microscope for imaging.

\subsection{Endothelial tube formation assay}

$50 \mu \mathrm{L}$ of Matrigel (BD Biosciences, Piscataway, NJ, USA) mixture (serum-free RPMl-1640 medium: Matrigel $=1: 1$ ) were added into 96 -well plate, and put it at $37^{\circ} \mathrm{C}$ until Matrigel was completely solidified. HUVECs $\left(1 \times 10^{5} /\right.$ well) were inoculated into 96-well plates containing Matrigel. Using microscope to collect microtubule formation pictures after $6 \mathrm{~h}$. 


\subsection{Flow cytometry assay}

THP-1 cells $\left(5 \times 10^{5} /\right.$ tube) were resuspended in the cell staining buffer after inducting to macrophages. THP- 1 cells were stained with APC-CD86, (Biolegend, San Diego, USA) for 20 min at $4{ }^{\circ} \mathrm{C}$. The cells washed with intracellular staining buffer. Added intracellular fluorescent staining with FITC-68 and PECD206, (Biolegend, San Diego, USA) for $20 \mathrm{~min}$ at $4{ }^{\circ} \mathrm{C} .500 \mu \mathrm{L}$ cell staining buffer was added to resuspend the cells, then analyzed with the BD Accuri flow cytometer (BD Bioscience, Piscataway, NJ, USA).

\subsection{Database assay}

We used Ualcan database (http://ualcan.path.uab.edu), CCLE database (https://portals.broadinstitute.org/ccle) and TIMER database (http://timer.comp-genomics.org) to analyze the expression of TMEM205 in cancer.

\subsection{Statistical assay}

GraphPad Prism 8.0 software (GraphPad, La Jolla, CA, USA) was used to data analysis. Two groups of data were compared by Student's $t$-test. Multiple groups of data were compared by One-way ANOVA. $P$ -

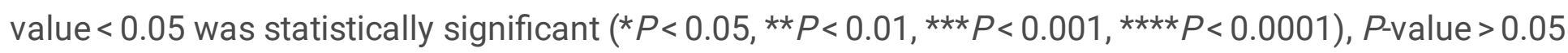
has no statistically significant (ns). All experiments were repeated three times.

\section{Results}

\subsection{DDP-resistant GC cells promote cancer stemness}

We detected the sensitivity of SGC-7901/DDP cells to DDP, and treated SGC-7901 and SGC-7901/DDP cells with different concentrations of DDP for $48 \mathrm{~h}$. MTT assay showed that the $\mathrm{IC}_{50}$ value of SGC-7901 cells is $0.9 \mu \mathrm{g} / \mathrm{mL}$, and the $\mathrm{IC}_{50}$ value of SGC-7901/DDP cells is $1.46 \mu \mathrm{g} / \mathrm{mL}$ (Fig. 1a). SGC-7901/DDP cells were more resistant to DDP compared with SGC-7901 cells. The colony formation ability of SGC7901/DDP cells were enhanced compared with SGC-7901 cells (Fig. 1b-C). Related studies showed that the migration, invasion and EMT progression of SGC-7901/DDP cells were enhanced compared with SGC-7901 cells ${ }^{[12-14]}$. In addition, we examined the effect of DDP-resistant GC on cancer stemness. Western blot assay showed that the expression of CD44, Oct4, Nanog and Sox2 were up-regulated in SGC-7901/DDP cells compared with SGC-7901 cells (Fig. 1d-e). IF assay showed that the fluorescence intensity of CD44 and Oct4 were enhanced in SGC-7901/DDP cells compared with SGC-7901 cells (Fig. 1f-g).

\subsection{DDP-resistant GC cells promote TAM/M2 polarization}


TAMs were associated with poor prognosis of cancer and involved in tumor angiogenesis and promoted tumor metastasis ${ }^{[15]}$. We detected the effect of DDP-resistant GC on TAM/M2 polarization in THP-1 cells of co-culture system (Fig. 2a). Western blot assay showed that SGC-7901/DDP cells up-regulated the expression of TAM/M2 marker CD206, and down-regulated the expression of TAM/M1 marker CD86 and iNOS in THP-1 cells compared with SGC-7901 cells (Fig. 2b-c). IF assay showed that SGC-7901/DDP cells enhanced the fluorescence intensity of CD206 and weakened the fluorescence intensity of CD86 in THP-1 cells compared with SGC-7901 cells (Fig. 2d-e). Flow cytometry assay showed that SGC-7901/DDP cells increased the percent of $\mathrm{CD} 206^{+} / \mathrm{CD} 68^{+}$cells, and decreased the percent of $\mathrm{CD} 86^{+} / \mathrm{CD} 68^{+}$cells in THP-1 cells compared with SGC-7901 cells (Fig. 2f-i). These results suggest that DDP-resistant in GC cells induced TAM/M2 polarization.

\subsection{TMEM205 enhances the proliferation and stemness of SGC-7901/DDP cells}

We found that TMEM205 overexpressed in GC through Ualcan, CCLE and TIMER databases (Fig. 3a-d). Western blot assay showed that TMEM205 overexpressed in SGC-7901/DDP cells compared with GES-1 cells and SGC-7901 cells (Fig. 3e-f). Then SGC-7901/DDP cells were transfected using si-Con and three different TMEM205 specific siRNA. Western blot assay showed that si-TMEM205-2 had the most obvious knockdown effect and was selected for the subsequent assays (Fig. 3g-h). We used si-TMEM205-2 and/or DDP $(1.46 \mu \mathrm{g} / \mathrm{mL})$ to detect the effect of TMEM205 on SGC-7901/DDP cell proliferation. MTT, EDU and clone formation assays showed that the combined treatment group significantly inhibited the proliferation of SGC-7901/DDP cells compared with the single treatment group (Fig. 4a-e). Western blot assay showed that the combined treatment group significantly down-regulated the expression of CD44, Oct4, Nanog and Sox2 compared with the single treatment group (Fig. 4f-g). IF assay showed that the combined treatment group significantly weakened the fluorescence intensity of CD44 and Oct4 in SGC7901/DDP cells compared with the single treatment group (Fig. 4h-i).

\subsection{TMEM205 promotes migration and EMT process of SGC-7901/DDP cells}

Metastasis was the main cause of poor prognosis of cancer, and angiogenesis was a necessary process for aggressive tumor growth and metastasis ${ }^{[16]}$. Thence we detected the effects of TMEM205 on SGC$7901 /$ DDP cells migration. Wound healing and transwell assays showed that the combined treatment group significantly inhibited the migration of SGC-7901/DDP cells compared with the single treatment group (Fig. 5a-d). Endothelial tube formation assay showed that the combined treatment group significantly inhibited the microtubule formation of HUVECs compared with the single treatment group (Fig. 5e-f). Western blot assay showed that the combined treatment group significantly down-regulated the expression of VEGF, MMP2 and MMP9 in SGC-7901/DDP cells compared with the single treatment group (Fig. 5g-h). 
The EMT process played important roles in tumor invasion, migration and angiogenesis ${ }^{[17]}$. Therefore, we detected the effect of TMEM205 on EMT process of SGC-7901/DDP cells. Western blot assay showed that the combined treatment group significantly up-regulated the expression of epithelial markers Ecadherin and ZO-1, and down-regulated the expression of mesenchymal markers Twist, N-cadherin, Vimentin, Slug and Snail in SGC-7901/DDP cells compared with the single treatment group (Fig. 5i-j). IF assay showed that the combined treatment group significantly enhanced the fluorescence intensity of Ecadherin and weakened the fluorescence intensity of Vimentin in SGC-7901/DDP cells compared with the single treatment group (Fig. 5k-I).

\subsection{TMEM205 induces TAM/M2 polarization}

We detected the effect of TMEM205 on the polarization of TAM/M2 in co-culture system. Western blot assay showed that the combined treatment group significantly down-regulated the expression of CD206 and up-regulated the expression of CD86 and iNOS in THP-1 cells compared with the single treatment group (Fig. 6a-b). Flow cytometry assay showed that the combined treatment group decreased the percent of $\mathrm{CD} 206^{+} / \mathrm{CD} 68^{+}$cells and increased the percent of $\mathrm{CD} 86^{+} / \mathrm{CD} 68^{+}$cells in THP- 1 cells compared with the single treatment group (Fig. 6c-f). IF assay showed that the combined treatment group significantly weakened the fluorescence intensity of CD206 and enhanced the fluorescence intensity of CD86 in THP-1 cells compared with the single treatment group (Fig. 6g-h).

\subsection{TMEM205 induces activation of Wnt/ $\beta$-catenin pathway in SGC-7901/DDP cells}

The Wnt/ $\beta$-catenin signaling pathway played active roles in promoting tumor formation, chemoresistance and stemness ${ }^{[18,19]}$. To detect the possible mechanism of TMEM205 in SGC-7901/DDP cells, we detected the effect of TMEM205 on Wnt/ $\beta$-catenin signaling pathway. Western blot assay showed that the combined treatment group significantly down-regulated the expression of Wnt/ $\beta$-catenin pathway related proteins Wnt, $\beta$-catenin and C-myc in SGC-7901/DDP cells compared with the single treatment group (Fig. $6 \mathrm{i}-\mathrm{j})$. IF assay showed that the combined treatment group inhibited the expression of $\beta$-catenin into nucleus of SGC-7901/DDP cells compared with the single treatment group (Fig. 6k).

\section{Discussion}

As the common malignant tumor, chemotherapy was the important treatment for $\mathrm{GC}^{[14]}$. And DDP was a more frequent choice of $\mathrm{GC}^{[2]}$. With the concentration of drugs increased, cancer cells gradually became resistant. Therefore, it is necessary to find a target that can reverse the drug resistance of GC to improve clinical benefit.

TMEMs were integral membrane proteins, which played important roles in the process of cell information transmission ${ }^{[3]}$. Related studies have found that TMEM205 overexpressed in DDP resistant ovarian cancer and participated in the therapeutic process of DDP for ovarian cancer ${ }^{[20]}$. TMEM88 overexpression inhibited cells proliferation, and enhanced the sensitivity of ovarian cancer to DDP ${ }^{[21]}$. These results 
indicated that TMEMs can affect the occurrence, development and chemoresistance of cancer. However, whether TMEM205 could affect the sensitivity of GC to DDP had no studies. Our study results showed that TMEM205 enhanced the resistance of GC to DDP by promoting the proliferation and stemness of SGC-7901/DDP cells.

Metastasis can increase cancer resistance to chemotherapy drugs ${ }^{[22]}$. Meanwhile, metastasis involved multiple processes and interrelated steps, such as angiogenesis, EMT, and changes in the tumor microenvironment ${ }^{[23]}$. DDP-resistant lung cancer cells promoted distant metastasis of lung cancer ${ }^{[24]}$. TMEM230 promoted the malignant process of glioblastoma by promoting tumor angiogenesis ${ }^{[25]}$. However, whether TMEM205 involved in cancer metastasis had no studies. Our results showed that TMEM205 promoted the migration of SGC-7901 cells, therapy accelerating the progression of GC.

EMT was the process by which epithelial cells acquire mesenchymal characteristics, and played important roles in the occurrence, invasion, metastasis, stemness and chemoresistance of cancer ${ }^{[26,27]}$. Studies have shown that TMEM229 overexpression inhibited cells migration and invasion by preventing EMT process of lung cancer ${ }^{[28]}$. Connexin43 overexpression reversed tamoxifen resistance by inhibiting EMT process ${ }^{[29]}$. Whether TMEM205 affected the progression of GC through the EMT process had not been reported. Our results showed that TMEM205 promoted the proliferation, stemness, migration and angiogenesis of SGC-7901/DDP cells by inducing the EMT process, thereby accelerating the malignant progress of GC.

TAMs involved in cancer proliferation, migration, invasion and chemoresistance ${ }^{[30]}$. The phenotypic transition of TAMs contributed to cancer progression. Studies have found that TAMs could affect chemoresistance and cancer progression ${ }^{[31]}$. Triptolide affected DDP resistance in ovarian cancer by inducing TAM/M2 polarization ${ }^{[32]}$. However, whether TMEM205 affected the resistance of GC to DDP by regulating the polarization of TAMs had no studies. Our study results showed that TMEM205 enhanced the resistance of GC to DDP by inducing TAM/M2 polarization.

Activation of Wnt/ $\beta$-catenin signaling pathway played important roles in tumor cell proliferation, stemness, migration and chemotherapy resistance ${ }^{[18,33]}$. The protein of $\beta$-catenin nuclear translocation could induce the activation of C-myc and CyclinD1, thereby activating the Wnt/ $\beta$-catenin signaling pathway ${ }^{[34]}$. Studies have found that the competitive combination of TMEM88 and disheveled inhibited the activation of the Wnt/ $\beta$-catenin pathway, thereby inhibiting the progression of triple-negative breast cancer ${ }^{[35]}$. However, whether TMEM205 could affect cancer progression by regulating the $\mathrm{Wnt} / \beta$-catenin pathway had not reported. Our study showed that TMEM205 promoted the proliferation, stemness, migration, angiogenesis and EMT process of SGC-7901/DDP cells by activating Wnt/ $\beta$-catenin.

Taken together, the results of this study indicated that knocking down TMEM205 combined with DDP inhibited the proliferation, migration, angiogenesis and EMT process of SGC-7901/DDP cells. In terms of mechanism, TMEM205 played an important role in DDP resistance and malignant progression of GC through Wnt/ $\beta$-catenin signaling pathway. 


\section{Declarations}

\section{Acknowledgments}

This study was supported by grants from the National Natural Science Foundation of China (No. 81960554, No. 82060554), the Department of Science and Technology of Jilin Province (No. $20220414087 \mathrm{GH})$.

\section{Ethics approval}

"All applicable international, national, and/or institutional guidelines for the care and use of animals were followed."

\section{Declaration of interest statement}

The authors declare that they have no known competing financial interests or personal relationships that could have appeared to influence the work reported in this paper.

\section{References}

1. Pawluczuk E, Lukaszewicz-Zajac M, Mroczko B. The Role of Chemokines in the Development of Gastric Cancer - Diagnostic and Therapeutic Implications. Int J Mol Sci. 2020; 21(22):8456.

2. Smyth EC, Nilsson M, Grabsch HI, et al. Gastric cancer. The Lancet. 2020; 396(10251):635-648.

3. Schmit K, Michiels C. TMEM Proteins in Cancer. A Review. Front Pharmacol. 2018; 9:1345.

4. Shen DW, Ma J, Okabe M, et al. Elevated expression of TMEM205, a hypothetical membrane protein, is associated with cisplatin resistance. Journal of Cellular Physiology. 2010; 225(3):822-828.

5. Shen DW, Gottesman MM. RAB8 enhances TMEM205-mediated cisplatin resistance. Pharm Res. 2012; 29(3):643-650.

6. Xu H, Dun S, Gao Y, et al. TMEM107 inhibits EMT and invasion of NSCLC through regulating the Hedgehog pathway. Thorac Cancer. 2021; 12(1):79-89.

7. Liu Y, Zheng Q, He G, et al. Transmembrane protein 215 promotes angiogenesis by maintaining endothelial cell survival. J Cell Physiol. 2019; 234(6):9525-9534.

8. Yang B, Wang F, Zheng G. Transmembrane protein TMEM119 facilitates the stemness of breast cancer cells by activating Wnt/beta-catenin pathway. Bioengineered. 2021; 12(1):4856-4867.

9. Pan Y, Yu Y, Wang X, et al. Tumor-Associated Macrophages in Tumor Immunity. Front Immunol. 2020; 11:583084.

10. Peiming, Zheng, Lei, Chen, et al. Exosomal transfer of tumor-associated macrophage-derived miR-21 confers cisplatin resistance in gastric cancer cells. J Exp Clin Cancer Res. 2017;36(1):53. 
11. He Z, Chen D, Wu J, et al. Yes associated protein 1 promotes resistance to 5 -fluorouracil in gastric cancer by regulating GLUT3-dependent glycometabolism reprogramming of tumor-associated macrophages. Arch Biochem Biophys. 2021; 702:108838.

12. Li P, Hu J, Shi B, et al. Baicalein enhanced cisplatin sensitivity of gastric cancer cells by inducing cell apoptosis and autophagy via Akt/mTOR and Nrf2/Keap 1 pathway. Biochem Biophys Res Commun. 2020; 531(3):320-327.

13. Li C, Zou J, Zheng G, et al. MiR-30a Decreases Multidrug Resistance (MDR) of Gastric Cancer Cells. Medical Science Monitor. 2016; 22:4509-4515.

14. Ge L, Li DS, Chen F, et al. TAZ overexpression is associated with epithelial-mesenchymal transition in cisplatin-resistant gastric cancer cells. Int J Oncol. 2017; 51(1):307-315.

15. Cheng N, Bai X, Shu Y, et al. Targeting tumor-associated macrophages as an antitumor strategy. Biochem Pharmacol. 2021; 183:114354.

16. Folkman JJSio. Role of angiogenesis in tumor growth and metastasis. Arch Biochem Biophys. 2021;702:108838.

17. Yang F, Shao C, Wei K, et al. miR-942 promotes tumor migration, invasion, and angiogenesis by regulating EMT via BARX2 in non-small-cell lung cancer. J Cell Physiol. 2019;234(12):23596-23607.

18. Zhang $Y$, Wang $X$. Targeting the Wnt/beta-catenin signaling pathway in cancer. J Hematol Oncol. 2020; 13(1):165.

19. Xu X, Zhang M, Xu F, et al. Wnt signaling in breast cancer: biological mechanisms, challenges and opportunities. Mol Cancer. 2020; 19(1):165.

20. Calo CA, Smith BQ, Dorayappan KDP, et al. Aberrant expression of TMEM205 signaling promotes platinum resistance in ovarian cancer: An implication for the antitumor potential of DAP compound. Gynecol Oncol. 2021;S0090-8258(21)01520-1.

21. de Leon $M$, Cardenas $H$, Vieth $E$, et al. Transmembrane protein 88 (TMEM88) promoter hypomethylation is associated with platinum resistance in ovarian cancer. Gynecol Oncol. 2016; 142(3):539-547.

22. Ma B, Wells A, Wei L, et al. Prostate cancer liver metastasis: Dormancy and resistance to therapy. Semin Cancer Biol. 2021;71:2-9.

23. Guan X. Cancer metastases: challenges and opportunities. Acta Pharm Sin B. 2015; 5(5):402-418.

24. Chen X, Lv Y, Xu K, et al. DCBLD2 Mediates Epithelial-Mesenchymal Transition-Induced Metastasis by Cisplatin in Lung Adenocarcinoma. Cancers (Basel). 2021;13(6):1403.

25. Cocola C, Magnaghi V, Abeni E, et al. Transmembrane Protein TMEM230, a Target of Glioblastoma Therapy. Front Cell Neurosci. 2021;15:703431.

26. Pastushenko I, Blanpain C. EMT Transition States during Tumor Progression and Metastasis. Trends Cell Biol. 2019; 29(3):212-226.

27. Ashrafizadeh M, Zarrabi A, Hushmandi K, et al. Association of the Epithelial-Mesenchymal Transition (EMT) with Cisplatin Resistance. Int J Mol Sci. 2020; 21(11):4002. 
28. Zhang $X$, He $Y$, Jiang $Y$, et al. TMEM229A suppresses nonsmall cell lung cancer progression via inactivating the ERK pathway. Oncol Rep. 2021; 46(2):176.

29. Wu DP, Zhou Y, Hou LX, et al. CX43 deficiency confers EMT-mediated tamoxifen resistance to breast cancer via c-Src/PI3K/Akt pathway. Int J Biol Sci. 2021;17(10):2380-2398.

30. Boutilier AJ, Elsawa SF. Macrophage Polarization States in the Tumor Microenvironment. Int J Mol Sci. 2021; 22(13):6995.

31. Qin T, Li B, Feng X, et al. Abnormally elevated USP37 expression in breast cancer stem cells regulates stemness, epithelial-mesenchymal transition and cisplatin sensitivity. J Exp Clin Cancer Res. 2018; 37(1):287.

32. Le F, Yang L, Han Y, et al. TPL Inhibits the Invasion and Migration of Drug-Resistant Ovarian Cancer by Targeting the PI3K/AKT/NF-kappaB-Signaling Pathway to Inhibit the Polarization of M2 TAMs. Front Oncol. 2021; 11:704001.

33. Colozza G, Koo BK. Wnt/beta-catenin signaling: Structure, assembly and endocytosis of the signalosome. Dev Growth Differ. 2021; 63(3):199-218.

34. Chatterjee A, Paul S, Bisht B, et al. Advances in targeting the WNT/beta-catenin signaling pathway in cancer. Drug Discov Today. 2021;S1359-6446(21)00317-2

35. Yu X, Zhang X, Zhang Y, et al. Cytosolic TMEM88 promotes triple-negative breast cancer by interacting with Dvl. Oncotarget. 2015; 6(28):25034-25045.

\section{Figures}




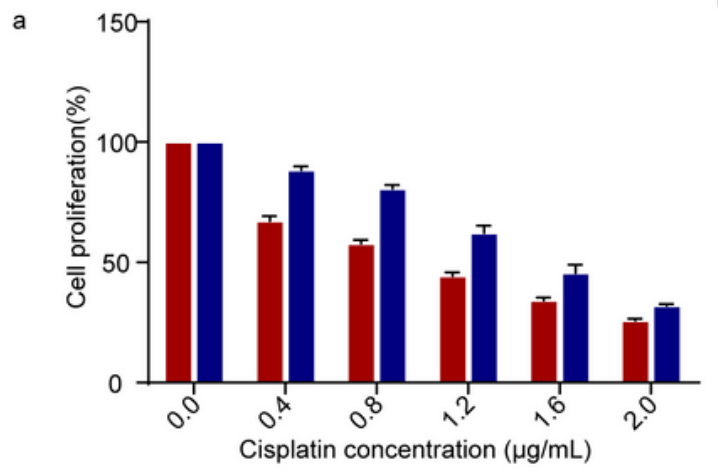

SGC-7901 + - + + + + + + +

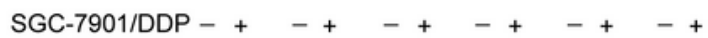

d

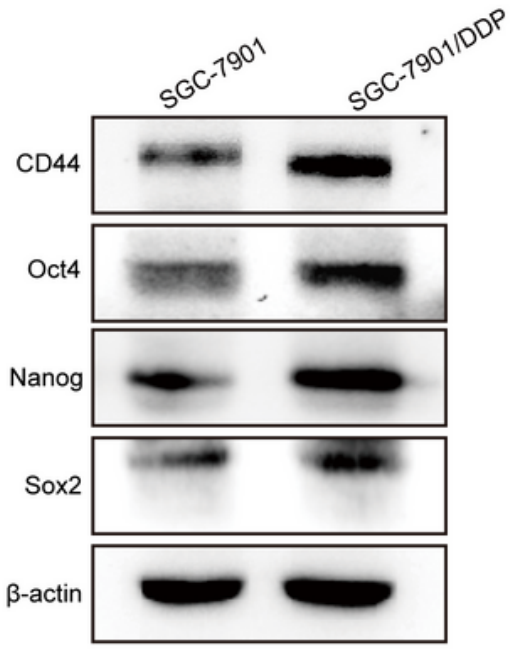

f

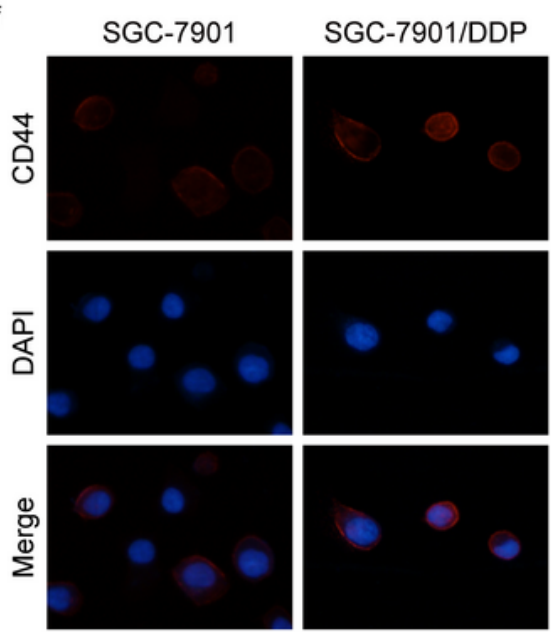

b SGC-7901 SGC-7901/DDP
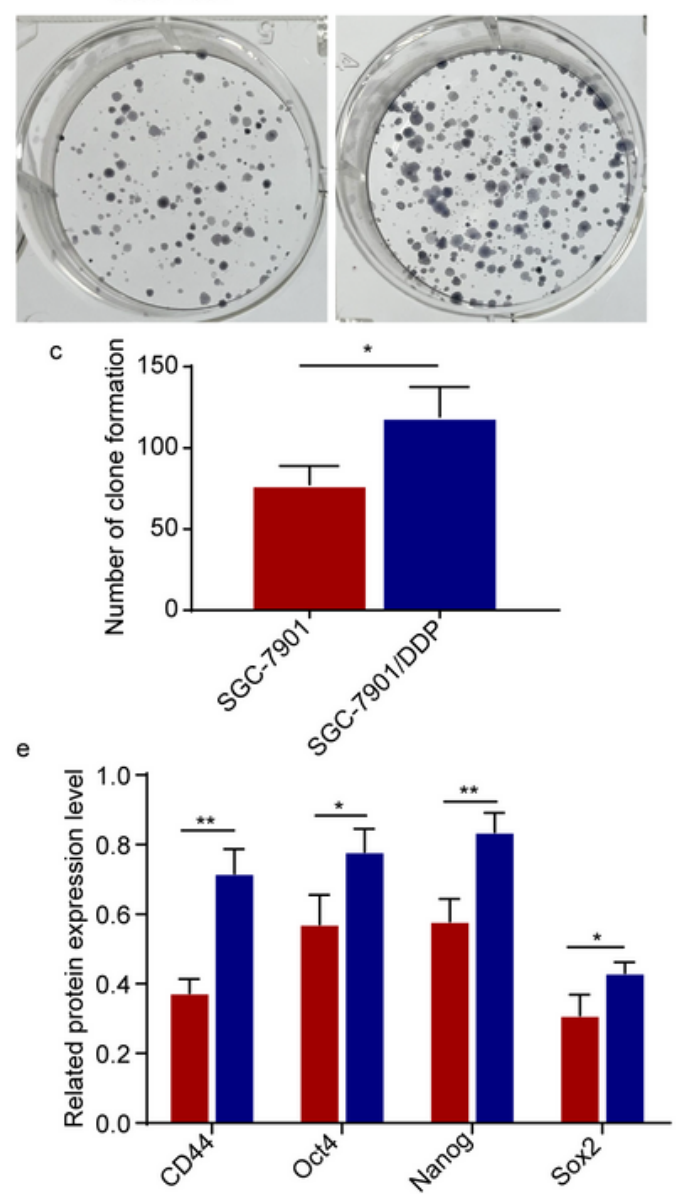

SGC-7901 + SGC-7901/DDP -

$\mathrm{g}$

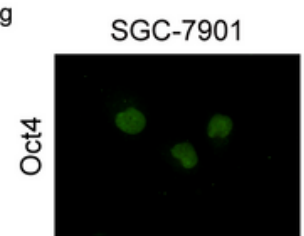

SGC-7901/DDP
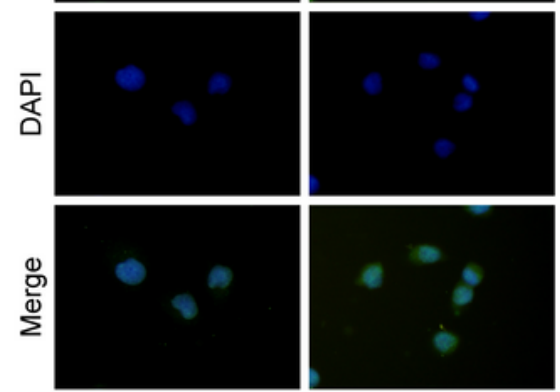

\section{Figure 1}

DDP-resistant GC cells promote cancer stemness. (a) MTT assay detected the effects of DDP on the proliferation of SGC-7901 and SGC-7901/DDP cells. (b-c) Colony formation assay detected the proliferation of SGC-7901 and SGC-7901/DDP cells. (d-e) Western blot assay showed the expression of CD44, Oct4, Nanog, and Sox2 in SGC-7901 and SGC-7901/DDP cells. (f-g) IF detected the fluorescence intensity of CD44 and Oct4 in SGC-7901 and SGC-7901/DDP cells (400×). 


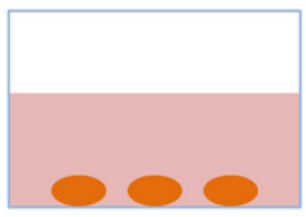

TAMs

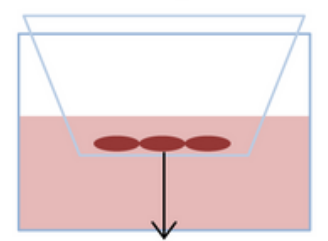

GC cells

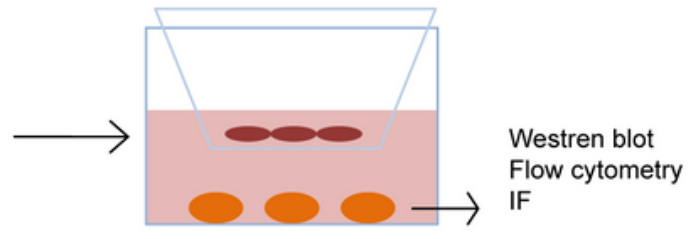

Flow cytometry
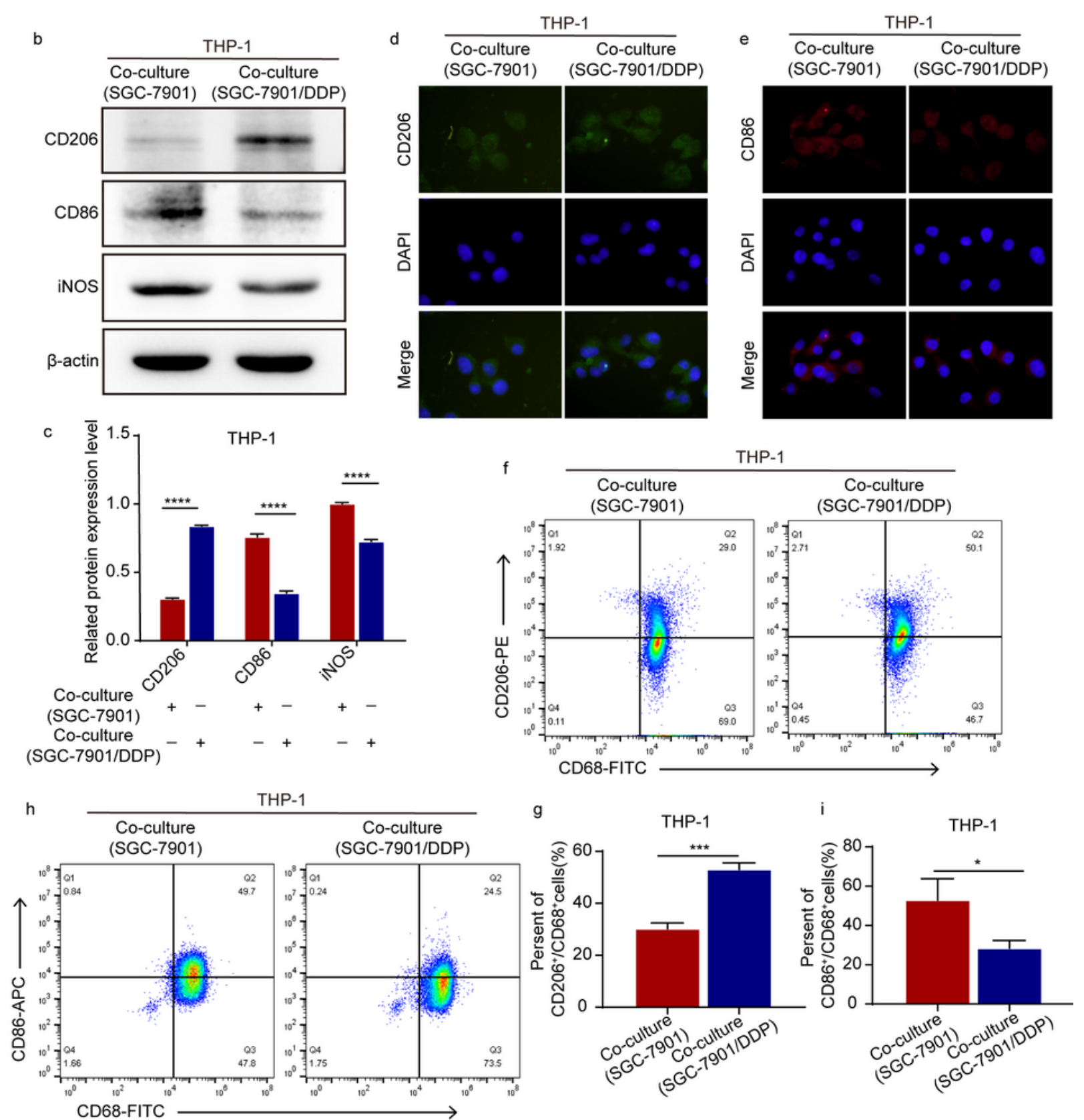

Figure 2

DDP-resistant GC cells induce TAM/M2 polarization. (a) Schematic diagram of co-culture system. (b-c) Western blot assay detected the expression of CD206, CD86 and iNOS in THP-1 cells of co-culture system. (d-e) IF detected the fluorescence intensity of CD206 and CD86 in THP-1 cells (400x). (f-i) Flow cytometry assay detected the percent of $\mathrm{CD} 206^{+} / \mathrm{CD} 68^{+}$cells and $\mathrm{CD} 86^{+} / \mathrm{CD} 68^{+}$cells in THP- 1 cells. 


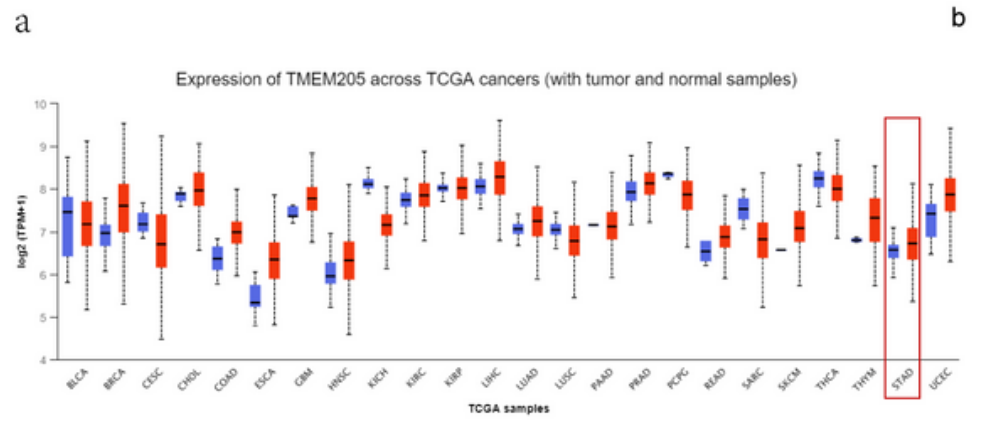

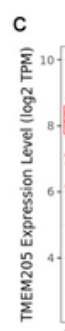

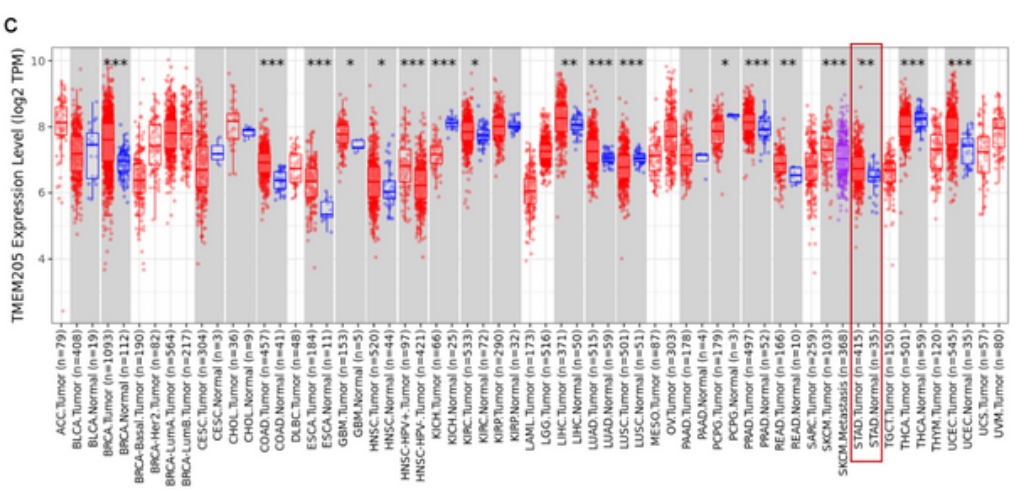

mRNA expression(RNAseq):TMEM205

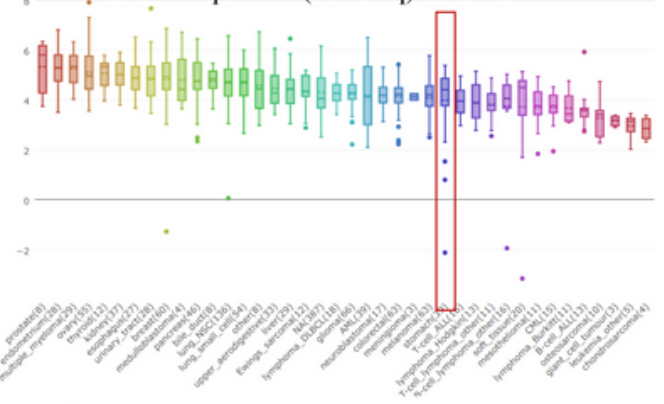

d

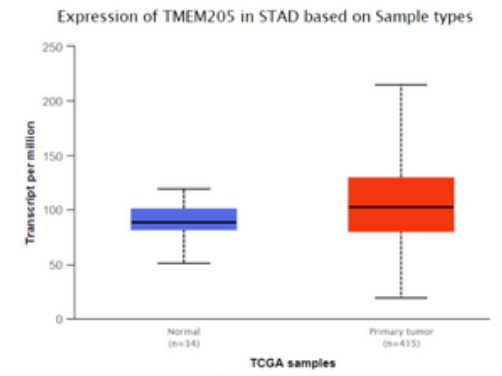

\begin{tabular}{c|c} 
Comparison & Statistical significance \\
\hline Normal-vs-Primary & $1.046499999776996 \mathrm{E}-08$
\end{tabular}

SGC-7901/DDP
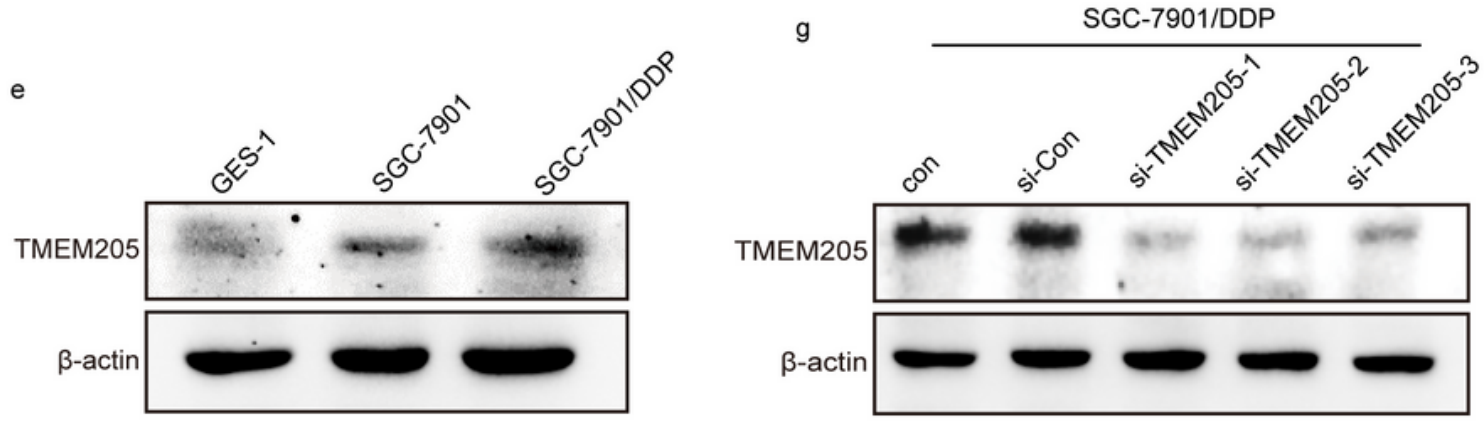

SGC-7901/DDP

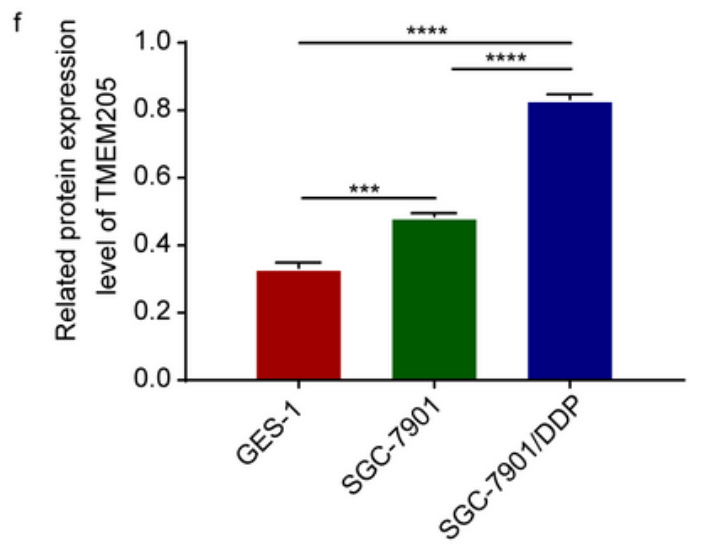

$\mathrm{h}$

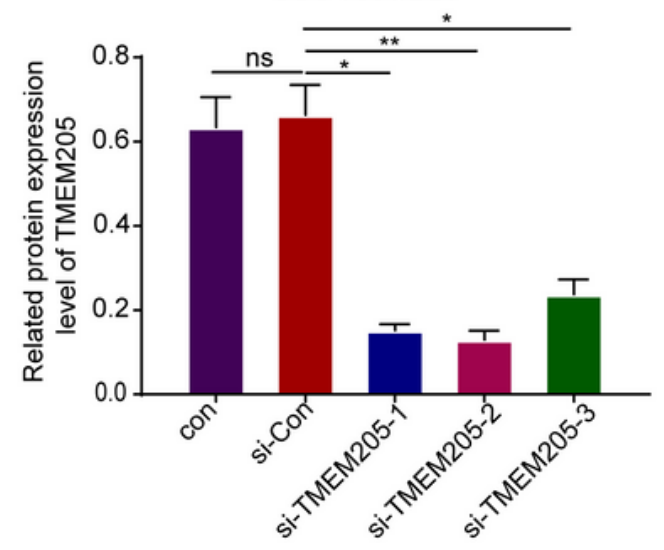

\section{Figure 3}

TMEM205 overexpressed in GC. (a-d) Ualcan, CCLE and TIMER databases detected the expression of TMEM205. (e-f) Western blot assay detected the expression of TMEM205 in GES-1, SGC-7901 and SGC7901/DDP cells. (g-h) Western blot detected the expression of TMEM205 in si-TMEM205-transfected SGC-7901/DDP cells. 

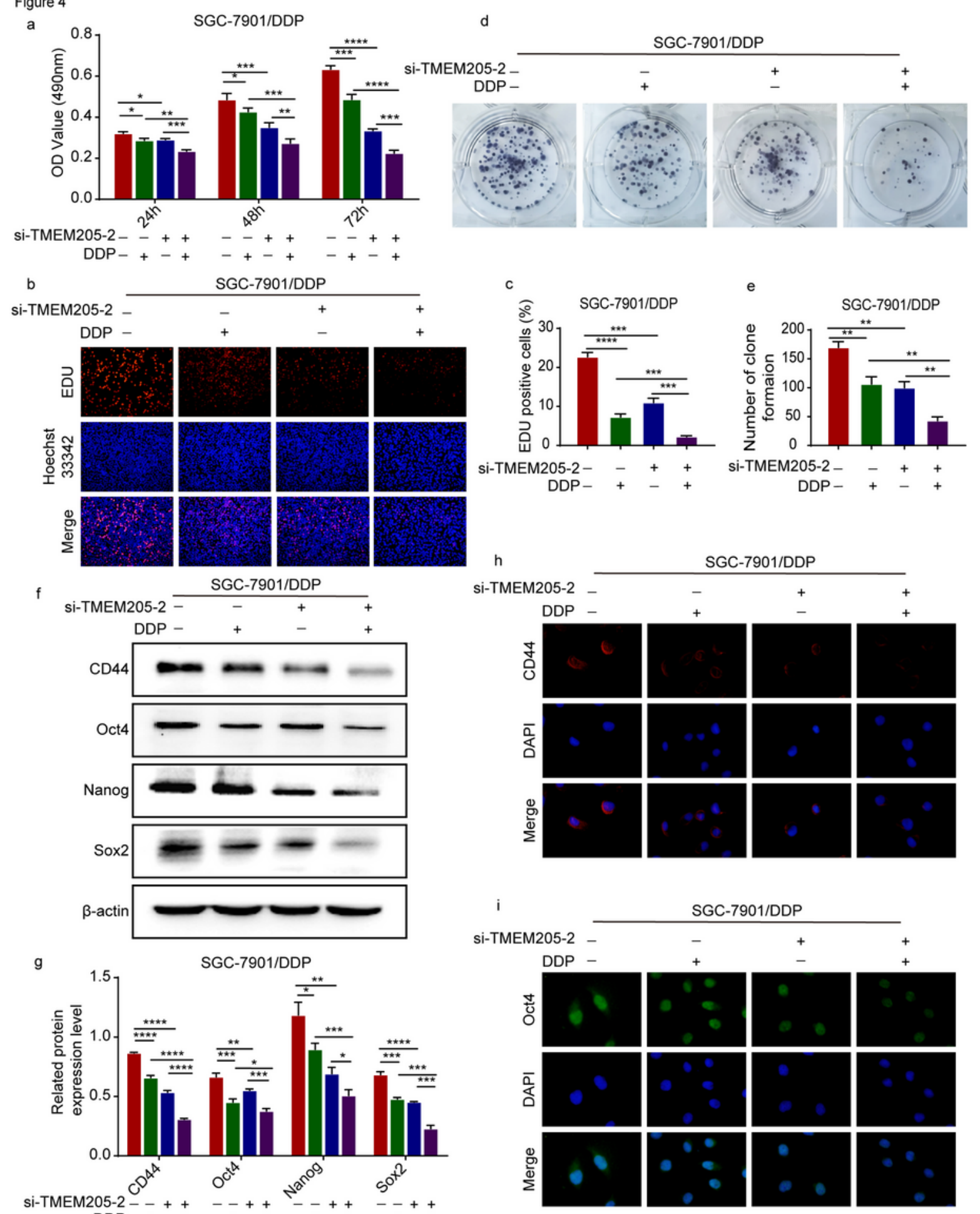

\section{Figure 4}

TMEM205 enhances the proliferation and stemness of SGC-7901/DDP cells. (a) MTT detected the proliferation of SGC-7901/DDP cells after using si-TMEM205-2 and/or DDP (1.46 $\mu \mathrm{g} / \mathrm{mL})$. (b-c) EDU assay detected the proliferation of SGC-7901/DDP cells of single or combined treatment groups. (d-e) Colony formation assay detected the proliferation of SGC-7901/DDP cells of single or combined treatment groups. (f-g) Western blot assay detected the expression of stemness marker (CD44, Oct4, 
Nanog and Sox2) in SGC-7901/DDP cells of single or combined treatment groups. (h-i) IF assay detected the fluorescence intensity of CD44 and Oct4 of SGC-7901/DDP cells of single or combined treatment groups (400x).
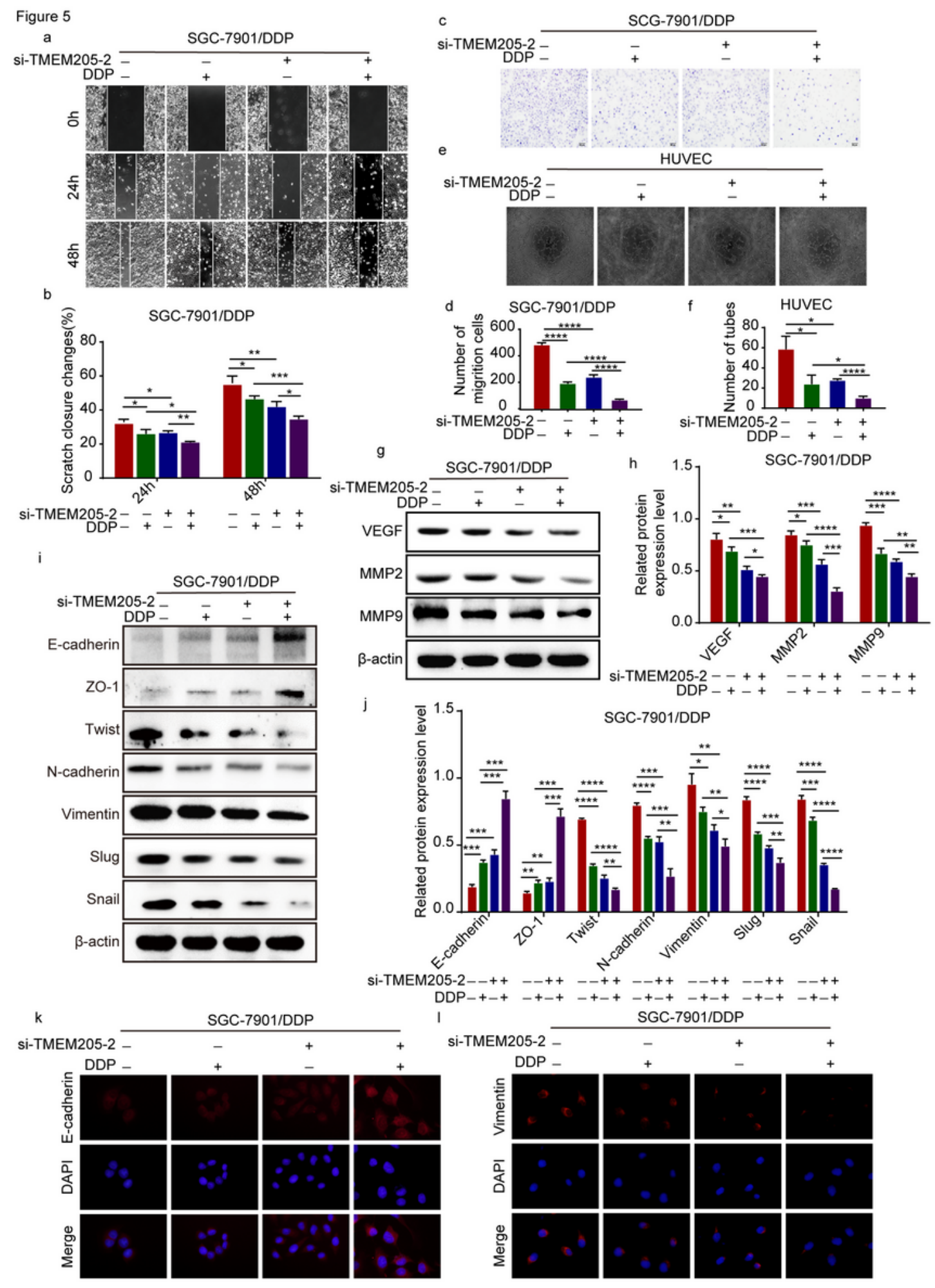

Figure 5 
TMEM205 promotes migration and EMT process of SGC-7901/DDP cells. (a-b) Wound healing assay detected the wound healing capacity of SGC-7901/DDP cells of single or combined treatment groups. (cd) Transwell assay detected the migration capacity of SGC-7901/DDP cells of single or combined treatment groups (200x). (e-f) Endothelial tube formation assay detected the microtubule formation of HUVECs of single or combined treatment groups (100x). (g-h) Western blot assay detected that the expression of VEGF, MMP2, and MMP9 in SGC-7901/DDP cells of single or combined treatment groups. (i-j) Western blot assay detected the expression of EMT-related markers of SGC-7901/DDP cells of single or combined treatment groups. ( $\mathrm{k}-\mathrm{I})$ IF assay detected the fluorescence intensity of E-cadherin and Vimentin of SGC-7901/DDP cells of single or combined treatment groups (400x). 


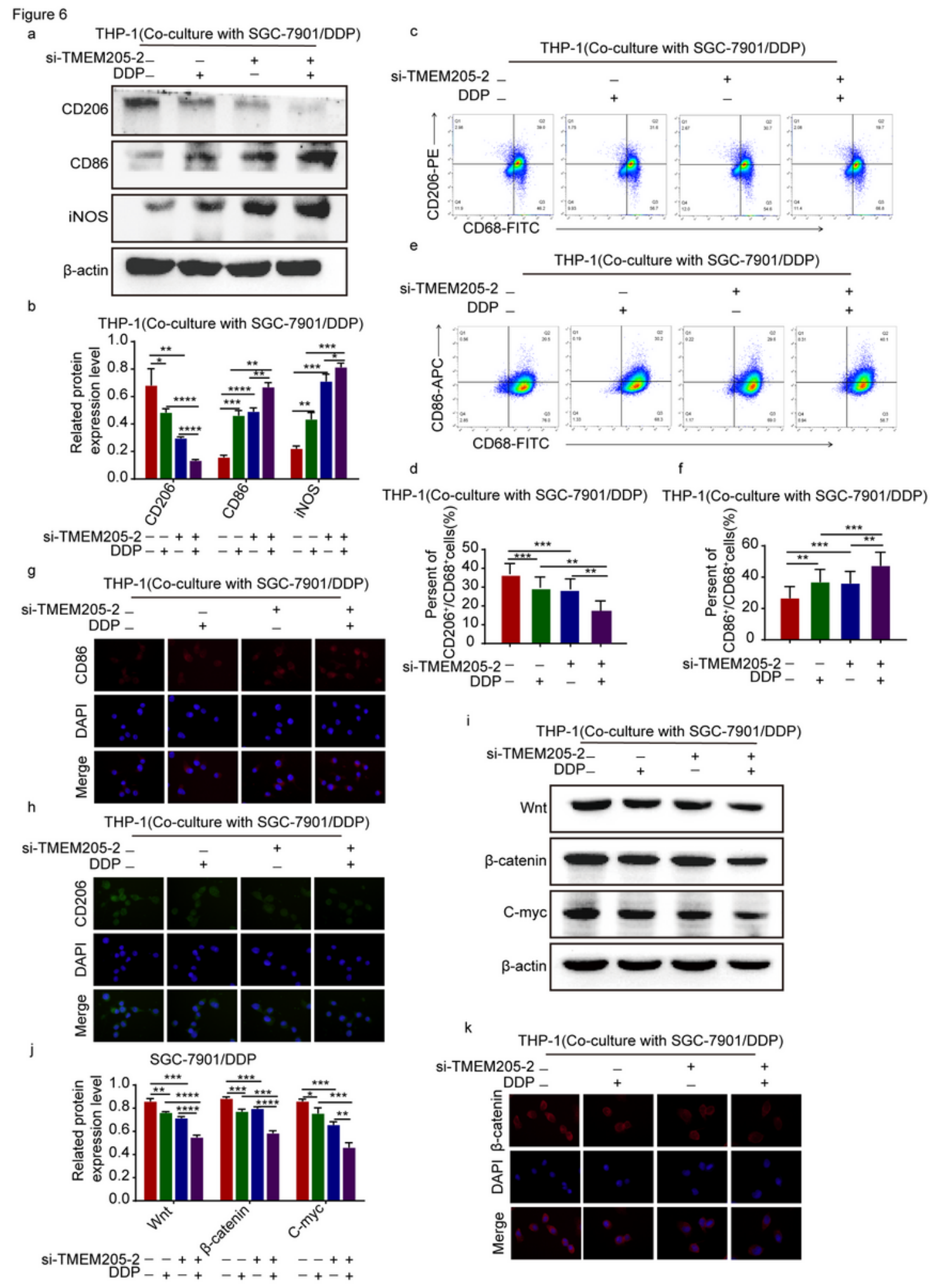

Figure 6

TMEM205 induces TAM/M2 polarization and activation of Wnt/ $\beta$-catenin pathway in SGC-7901/DDP cells. (a-b) Western blot assay showed the expression of CD206 and CD86 in THP-1 cells of co-culture system of single or combined treatment groups. (c-f) Flow cytometry assay detected the percent of CD206 ${ }^{+} / \mathrm{CD} 68^{+}$cells and CD $86^{+} / \mathrm{CD} 68^{+}$cells in THP- 1 cells. (g-h) IF assay detected the fluorescence intensity of CD206 and CD86 in THP-1 cells. (i-j) Western blot assay showed the expression of Wnt/ $\beta$ - 
catenin pathway related proteins of SGC-7901/DDP cells of single or combined treatment groups. (k) IF detected the fluorescence intensity of $\beta$-catenin in SGC-7901/DDP cells of single or combined treatment groups.

\section{Supplementary Files}

This is a list of supplementary files associated with this preprint. Click to download.

- graphicalabstract.png 